\title{
Diver's hand: a skin disorder common in occupational saturation diving
}

\author{
Catrine Ahlén, Ole Jan Iversen, Jan Risberg, Gunnar Volden, Harald Aarset
}

\begin{abstract}
Saturation divers regularly inspect North Sea installations, working at depth for periods of 12-16 days. Diver's hand is a particular problem in saturation diving, and there is no effective protection or treatment available. This paper presents the occurrence of diver's hand and describes the disease in clinical and epidemiological terms. Three studies of diver's hand have been carried out, in 1990, 1994, and 1995. Most long term saturation divers have had diver's hand at some time in their professional career. Diver's hand seems able to occur without any previous skin symptoms, and divers without diver's hand can have several other skin symptoms during a saturation period. It is likely that diver's hand is a specific phenomenon associated with saturation diving.
\end{abstract}

(Occup Environ Med 1998;55:141-143)

Keywords: palms; skin syndrome; saturation diving

Saturation diving has been extensively used for maintenance and inspection of North Sea offshore subsea oil installations. Typically, the divers live in steel chambers in an atmosphere of pressurised helium and oxygen. ${ }^{1}$ The working period includes compression lasting one to three hours, bottom time (working period) lasting 12-16 days, and decompression lasting four to seven days. When working, the divers will dress in a protective diving suit, in which heated $\left(\sim 36^{\circ} \mathrm{C}\right)$ sea water is continuously flushed on to the divers' skin to maintain thermal balance. Respiratory heat loss is considerable during deep diving as helium has a thermal conductivity about six times higher than air, and respiratory heat loss increases proportionally to gas density ( depth). Such a "bell run" may last for six hours, whereas the working period outside the bell "lock out" may last for four hours for the individual diver. The ambient pressure is dependent on the working depth, which normally in the North Sea is 50-200 m, 0.6-2.1 MPa. The partial pressure of oxygen will vary between 20 to $50 \mathrm{kPa}$ depending on the phase of the dive. The ambient temperature in the chambers varies with the working depth, but will often be around $28-30^{\circ} \mathrm{C}$. The relative humidity is generally high and may reach $80 \%-90 \%$, but is normally set to be $<50 \%$.

Skin infections caused by Pseudomonas aeruginosa are common among occupational divers in the North Sea. ${ }^{2}$ External otitis is well known, and may become severe when in saturation. ${ }^{3}$ Less known are other superficial skin infections of the face and neck. ${ }^{4}$

Another common skin disorder among saturation divers appears as extensive skin peeling of the upper skin layers of the palms of the hands and occasionally of the soles of the feet. This disorder is referred to as "diver's hand" by the divers. ${ }^{5}$ It is a considerable medical problem for saturation divers, and so far, no effective protection or treatment is available.

The objectives of this paper are to present the occurrence of diver's hand among saturation divers and to describe the disease in clinical and epidemiological terms.

\section{Subjects and methods}

Between 1990 and 1995, three separate studies on diver's hand have been performed, study 1 in 1990, 2 in 1994, and 3 in 1995. All divers studied volunteered to participate.

STUDY 1

The main objective of the initial study was to map the prevalence of diver's hand among saturation divers. A questionnaire was submitted to 120 occupational divers belonging to a single diving contractor. The questionnaire focused mainly on the divers' personal experiences of the skin problem termed diver's hand. No attempt was made to define criteria for diver's hand in this study.

STUDY 2

The main objective was to obtain a clinical description of diver's hand, based on clinical examination, interview, and histological analyses of skin biopsies. Twelve divers participated in this study. Before compression, and immediately after decompression, the divers were examined by a dermatologist. During the observation period, each diver completed a daily questionnaire (daily $\log$ ) in which he was asked to report development of any skin symptom. The following symptoms were registered: redness, whiteness, itchiness, dryness, cracking, tightness and stiffness, swelling, scaling, peeling, soreness, and pins and needles. The symptoms were graded: not at all (0), little (1), moderate (2), and a lot (3). Also, the divers were asked to give information about other symptoms if present.

After completed decompression, two skin biopsies were taken from each diver for histological examination (one from an area of intensive peeling and one from the boundary between normal skin and peeled skin). The biopsies were conserved in $4 \%$ formalin, embedded in paraffin, and $5 \mu$ thick sections were stained with 


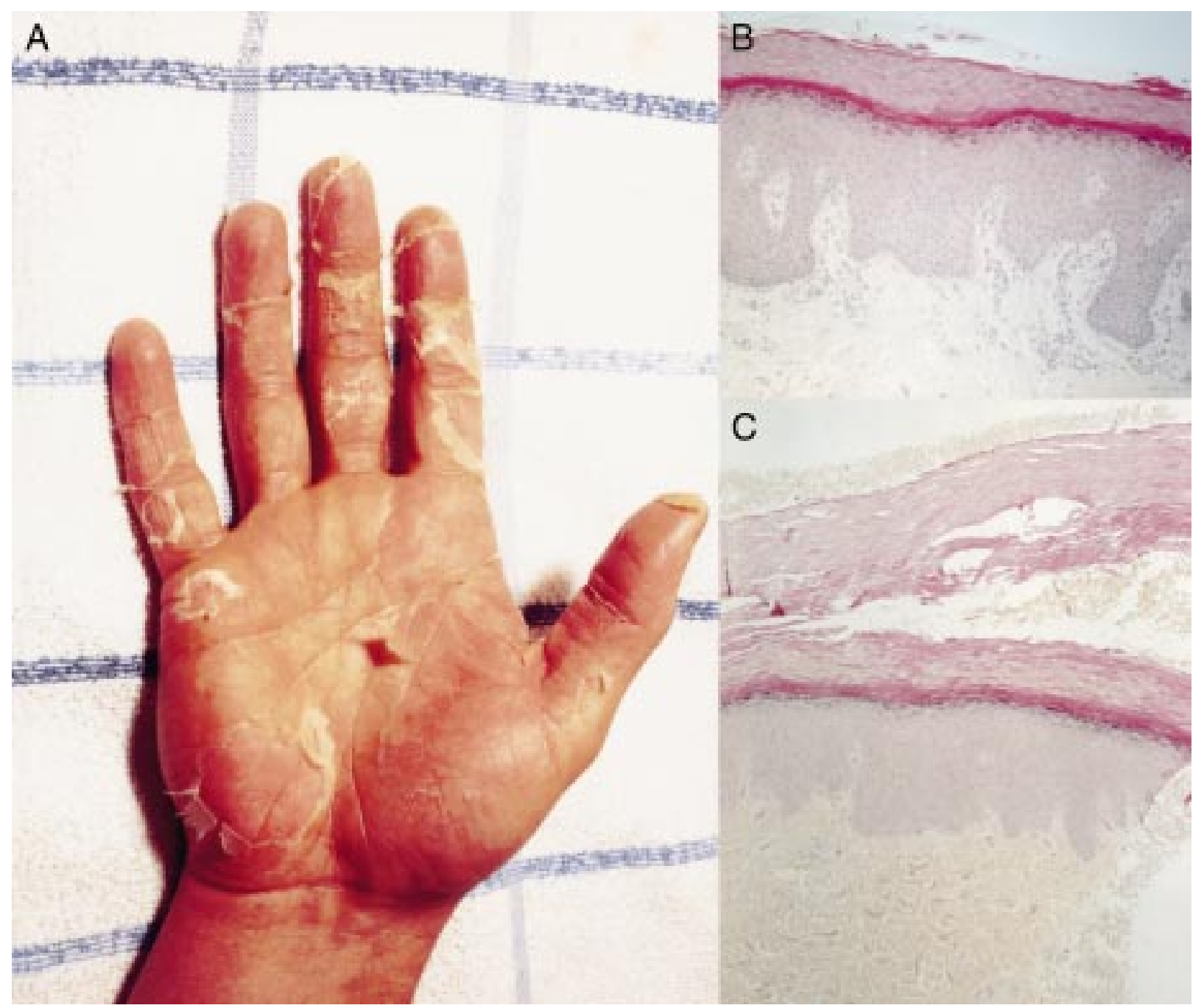

(A) A fully developed diver's hand. (B) A fully developed diver's hand, in which the upper two thirds or more of the stratum corneum has fallen off (haematoxylin-eosin-saphronin, originally $\times 200$ ). (C) Evolving diver's hand, with typical clefts in the upper two thirds of the stratum corneum (haematoxylin-eosin-saphronin, originally $\times 200$ ).

haematoxylin-eosin-saphronin, periodic acid Schiff (PAS), and Grocott stains.

STUDY 3

The main objective was to evaluate overall occurrence of skin symptoms on the palms of the hands among saturation divers, based on our knowledge from studies 1 and 2, and irrespective of earlier experience of diver's hand. The observation period was limited to the period of saturation. Thirty six divers participated in this study, all of them with a saturation period of more than 10 days.

\section{Results}

The first questionnaire (study 1) was completed and returned by 98 divers, $(82 \%)$. Of these, 81 divers $(83 \%)$ reported that they had experienced diver's hand at some time during their diving career. In the cohort with $>10$ years of offshore diving, the prevalence of diver's hand was $97 \%$. Thirty five per cent had at some time experienced similar problems in the feet (planta). These symptoms were experienced by $89 \%$ only during diving.

All divers in study 2 claimed to have had diver's hand, although none before their first saturation, despite a long onshore diving career. Most divers described several skin symptoms associated with the skin peeling. The development of diver's hand was reported to occur at any time during a saturation dive as well as after decompression.

The daily logs in studies 2 and 3 confirmed that diver's hand indeed may start during the bottom phase as well as during decompression, applying the criteria that either skin peeling of any degree or skin scaling that scored $\geqslant 2$ was required to define the skin symptoms as diver's hand. In the field study (study 3), we found that $50 \%$ of the divers developed diver's hand during the saturation period. Most of these 18 divers developed diver's hand before day 13 of saturation (day 0 included). The scaling and peeling off (diver's hand) occurred irrespectively of the presence of any other skin symptoms (data not shown). Considerable variations in occurrence of symptoms relative to diving site were registered (data not shown).

DIVER'S HAND: CLINICAL DESCRIPTION

All divers claiming to have had diver's hand reported peeling as the main complaint. Some divers experience mild scaling of small $1-5 \mathrm{~mm}$ 
pieces, but characteristically, large flakes of 3-6 $\mathrm{cm}^{2}$ of superficial skin can be peeled off. The scaling generally starts at the fingers and continues proximally. Palmar fissures are common. There are no vesicles or papulae. Hyperkerathosis is uncommon as are secondary infections. Figure A shows a typical diver's hand.

The scaling continues for one to two weeks. After this period, active scaling is usually finished, but the diver may complain of sore and thin skin as well as fissures for another two to four weeks. Some patients have additional symptoms of the hands even during initial stages of the disease - namely, pain, soreness, a burning sensation, a feeling of heat deep within the hand, or tight skin - although these symptoms are highly variable within and between the divers.

HISTOLOGICAL DESCRIPTION

Skin biopsies, taken from affected divers, showed formation of horizontal clefts in the stratum corneum. The lower third of the stratum corneum was mostly intact and showed no clefts, whereas the upper two thirds of the stratum corneum showed several clefts of varied lengths. Figure B shows that the margins of the clefts were irregular as if the stratum corneum had been pulled apart. In some biopsies the upper part of the stratum corneum had fallen off. Figure $\mathrm{C}$ shows that the distance from the stratum lucidum to the lowest of the clefts or top of the stratum corneum when the upper part had fallen off was measured as 0.3 $0.7 \mathrm{~mm}$. The clefts were larger and more numerous than artificial clefts usually seen in skin biopsies fixed with formalin and embedded in paraffin. The stratum malpighium and dermis did not show any pathological changes, especially no signs of inflammation. Special stains for fungi were negative.

\section{Discussion}

Diver's hand is a term used for an occupational skin disorder, characterised by extensive scaling and peeling of the skin on the palms. We have studied the occurrence of diver's hand among occupational saturation divers in the northern part of the North Sea. The high prevalence of diver's hand among saturation divers combined with the clinical consequences, make this disorder a substantial problem for these workers. Development of diver's hand is reported to occur in all phases of saturation (compression, bottom time, and decompression) as well as after the end of saturation.
The present work was restricted to off shore saturation divers employed in the British or Norwegian sector only, but does not imply a geographical localisation of the disease.

The clinical description of diver's hand indicates that this syndrome differs from other known skin diseases. Based on histological analyses, diver's hand seems to be caused by generation of clefts in the upper two thirds of the stratum curium. The absence of inflammatory components and infectious organisms could indicate that physical or chemical factors may be of aetiological relevance.

Based on our studies, we conclude that decompression might be of little or no relevance for development of diver's hand.

Diver's hand has occasionally resulted in loss of a diver's licence, but the main problems reported are that the divers feel socially ill at ease when returning with scaly and fissured hands to a spouse and children. They complain of increased sensitivity to non-specific toxic agents-such as solvents, petroleum products, etc, and manual work may be significantly hampered by pain in the hands.

The divers have described several different skin symptoms which they associate with diver's hand. The data obtained from the daily $\log$ do not support such a relation. Thus, we conclude that diver's hand can occur without any previous skin symptoms, and divers without diver's hand can have several skin symptoms other than extensive scaling or peeling during a saturation period. To our knowledge, this skin syndrome has not been described earlier, and is therefore probably a specific phenomenon associated with saturation diving.

We thank Statoil, Norsk Hydro, Saga Petroleum, and the Norwegian Petroleum Directorate for financial support. We also thank all the participating divers and the health personnel on board the diving vessels for their most skilful cooperation.

1 Freitag M, Woods A. Commercial diving: reference and operations handbook. Bath, Avon: John Wiley 1983;5:67-80.

2 Norwegian Petroleum Directorate. Report from the dive data base. Stavanger, Norway: Graphic Centre, NPD, 1995.

3 Alcock SR. Acute otitis externa in divers in the North Sea. A microbiological survey of seven saturation dives. fournal of Hygiene (Cambridge) 1977;78:395-409.

4 Ahlen C. FUDT: bacteriology 1988-90: a summary. Trondheim, Norway: SINTEF, 1991. (SINTEF report STF23 heim, Norway: SINTEF, 1991. (SINT)

5 Ahlen C, Brubakk AO, de Francisco P, et al. Diver's hand: a skin disorder in operational saturation divers. Proceeding XVIIth annual meeting of the European Underwater Biomedical Society (EUBS). Thessaloniki, Greece: EUBS, 1991: $493-8$ 